\title{
Comprehensive Analysis of Large Sets of Age-Related Physiological Indicators Reveals Rapid Aging around the Age of 55 Years
}

\author{
Erin Lixie Jameson Edgeworth Lior Shamir \\ Lawrence Technological University, Southfield, Mich., USA
}

\section{Key Words}

Physiological aging $\cdot$ Chronological aging $\cdot$ Aging

biomarkers · Population studies · Age-related changes

\begin{abstract}
Background: While many studies show a correlation between chronological age and physiological indicators, the nature of this correlation is not fully understood. Objective: To perform a comprehensive analysis of the correlation between chronological age and age-related physiological indicators. Method: Physiological aging scores were deduced using principal component analysis from a large dataset of 1,227 variables measured in a cohort of 4,796 human subjects, and the correlation between the physiological aging scores and chronological age was assessed. Results: Physiological age does not progress linearly or exponentially with chronological age: a more rapid physiological change is observed around the age of 55 years, followed by a mild decline until around the age of 70 years. Conclusion: These findings provide evidence that the progression of physiological age is not linear with that of chronological age, and that periods of mild change in physiological age are separated by periods of more rapid aging.

(c) 2015 S. Karger AG, Basel
\end{abstract}

(C) 2015 S. Karger AG, Basel

0304-324X/15/0616-0526\$39.50/0

\section{Introduction}

While physiological age correlates with chronological age, the correlation is clearly not consistent, and physiological aging varies across individuals. As no single aging biomarker that can reliably reflect aging has yet been discovered $[1,2]$, numerous studies have attempted to correlate specific physiological indicators with chronological age in populations of subjects, and they showed a significant correlation [2-6]. However, while some studies suggest that the correlation is linear, in many cases a close look at the data shows that while the correlation exists, it is not necessarily linear in all cases. An example is the work of Ma et al. [7], who studied the correlation between aging, insulin secretion and the disposition index. While the researchers suggest that the correlation is linear, the data show that a decline is observed from maturation to around the age of 45 years, after which stabilization sets in until after the age of 55 years, followed by a renewed decline [7].

The possible existence of distinct stages in aging can provide evidence that aging is driven also by biological pathways and not solely by stochastic accumulation of irreparable environmental damage. Distinct stages in the process of aging were observed in simpler organisms such as Caenorhabditis elegans by using analysis of age-regulated gene expression [8] as well as analysis of the progres-

\section{KARGER 125}

E-Mail karger@karger.com www.karger.com/ger

\footnotetext{
Lior Shamir

Lawrence Technological University

21000 West Ten Mile Road

Southfield, MI 48075 (USA)

E-Maillshamir@mtu.edu
} 
Table 1. Age distribution of the female and male participants from the OAI cohort

\begin{tabular}{llllllll}
\hline & $\begin{array}{l}45-50 \\
\text { years }\end{array}$ & $\begin{array}{l}50-55 \\
\text { years }\end{array}$ & $\begin{array}{l}55-60 \\
\text { years }\end{array}$ & $\begin{array}{l}60-65 \\
\text { years }\end{array}$ & $\begin{array}{l}65-70 \\
\text { years }\end{array}$ & $\begin{array}{l}70-75 \\
\text { years }\end{array}$ & $\begin{array}{l}75-80 \\
\text { years }\end{array}$ \\
\hline Women & 290 & 487 & 436 & 522 & 446 & 389 & 234 \\
Men & 259 & 380 & 359 & 241 & 254 & 297 & 202 \\
\hline
\end{tabular}

sion of tissue entropy [9]. These indicators show that the physiological aging of C. elegans is not linear with chronological age. Here, we use a large set of physiological, clinical, and environmental indicators collected from a large population of human subjects to synthesize a composite aging score, and show that the physiological indicators that correlate with aging do not progress in a linear fashion but instead feature several distinct stages.

\section{Design and Methods}

The data used in this study are taken from the Osteoarthritis Initiative (OAI) cohort [10], which is a comprehensive population study of an aging-related disease (osteoarthritis). The OAI dataset includes physiological variables such as weight and blood pressure, clinical indicators such as medications and medical history, and environmental indicators such as nutrition. All indicators were measured from all participants in the cohort. The full list of variables and their description is available on the $\mathrm{OAI} \mathrm{Web}$ site (http://oai.epiucsf.org/datarelease/SASDocs/VG_Variable. pdf). While no single variable is expected to reliably reflect physiological aging, it is assumed that at least some of these variables reflect aging and therefore the combination of all of these variables can sample physiological aging to provide a composite aging score as well as a quantitative approximation of the physiological aging state.

The dataset includes 4,796 individuals; 2,804 are women and 1,992 are men. The age distribution of the male and female participants is specified in table 1 . Two analysis methods were used to profile the progression of physiological aging. The first method is based on principal component analysis (PCA), which takes large quantities of correlated data and reduces them to a defined number of components [11]; it was also applied to predict mortality in humans [12]. The OAI data were reduced by PCA to six key components based on the categories of the OAI dataset [10]: physical activity, mental health, nutrition, medications, body type, and heart health. The categories and the number of variables in each category are displayed in table 2 .

The physical activity component comprised two subcategories: surveys and workouts. The patients took three surveys, and the scores of the surveys were used. Those surveys were the Medical Outcomes Study (MOS)-12 Short Form instrument, the Physical Activity Scale for the Elderly, and a personal survey about how much physical activity is involved in the participants' daily lives. The rest of the data were speeds and paces from several different time trials and endurance activities such as chair stands.

Aging Profile Analysis Using

Comprehensive Sets of Variables
Table 2. The principle components used to measure physiological aging

\begin{tabular}{lc}
\hline Component & Number of variables \\
\hline Physical activity & 35 \\
Mental health & 2 \\
Nutrition & 84 \\
Medications & 36 \\
Body type & 3 \\
Heart health & 3 \\
\hline
\end{tabular}

The mental health principal component was deduced from the variables collected in two surveys filled out by the OAI participants. These surveys are the Center for Epidemiologic Studies Depression Scale and the MOS-12 instrument. The nutrition component was the result of a self-assessment carried out by the participants. The survey asked about the daily content of their diet in terms of vitamins, minerals such as zinc and iron, and supplements.

The medications principal component was measured from a survey of almost 40 various prescriptions and supplements currently taken. The prescriptions included were salicylic acid, celecoxib, and aspirin among others. The body type principal component is made up of three variables: height, weight, and BMI. The heart health component is composed of average pulse, which has been shown to be an informative survival predictor at old age [12], as well as diastolic and systolic blood pressure. The six principal components described above were also reduced to a single component that expresses the physiological age of a person as reflected by the combination of physical activity, mental health, nutrition, medications, body, and heart health.

The downside of the cross-sectional analysis described above is that it might be biased by environmental effects having occurred in different years. Therefore, we also performed a longitudinal analysis of the principal components of aging, which is considered more reliable for the quantitative profiling of aging [13]. To accomplish this, we used two sets of the OAI data. The first set is derived from an initial visit, including data from 4,796 patients with measures of 1,226 variables. The second set stems from a follow-up visit 72 months after the initial visit. Only variables present in both datasets were used in this study, so that the values for each variable at these two distinct time points could be compared. This left 678 variables available for the analysis.

For each variable, the mean values and mean differences for each age were calculated and stored in new arrays. For each age, 
Fig. 1. Physiological aging score and standard error of the mean of the physiological aging scores at different chronological ages.

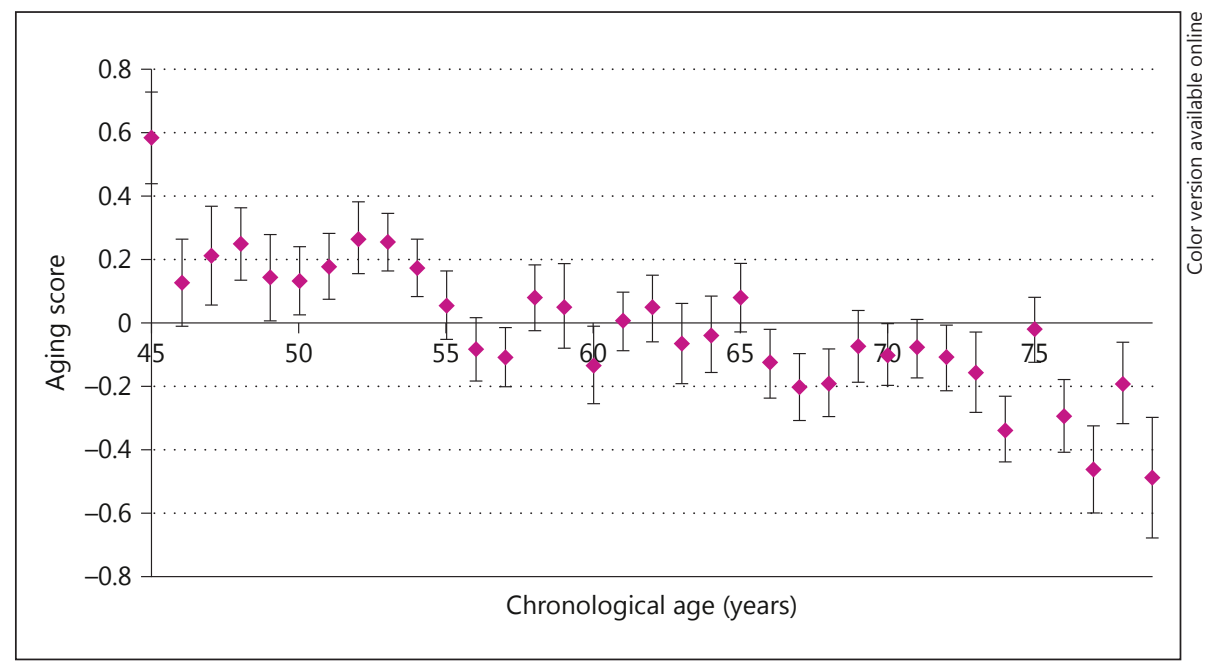

the values of the variable before and after that age were recorded, and the mean difference for each variable at each age was computed. The differences between the values before and after each age were also used to perform the PCA.

The PCA was used to create the different composite aging scores described above from the mean differences over the 72 -month time span for groups of related variables. The scores we computed included a heart health score, a weight/BMI score, a medications score, a mental health score, and a physical activity score. A total PCA of all these scores combined was conducted as well. We calculated these scores for the entire population of subjects, as well as for males and females separately.

In addition to the PCA, we also applied a method for computing the composite aging score using the correlation of each variable with chronological age. All values for each of the 1,227 variables were normalized to a mean of 25 and a standard deviation of 5 . Normalization for the standard deviation does not change the profile of change in a given variable across different ages, but it normalizes the magnitude of the change, so that different variables can be combined into a single composite score. For instance, if the range of a certain variable is $0-1$ and the range of another variable is $0-100$, changing the mean of both variables to 25 will not allow combining the two variables, because the variable with the range $0-100$ will have a stronger impact on the composite score than the variable with the smaller range.

Since not all variables correlate with chronological age (e.g., height), the Pearson correlation of each variable with chronological age was computed, and the variables of which the Pearson correlation with age was between -0.05 and 0.05 were rejected. Rejecting the variables with a weak or no correlation with chronological age provided a set of 329 variables. The composite aging score for each subject could be determined by the mean of the 329 variables.

One limitation of the described method is that it does not account for differences in the strength of a correlation within the set of variables that met the correlation criteria of 0.05 . That is, a variable of which the correlation with age is 0.6 will have the same effect on the composite aging score as a variable with a weaker correlation with chronological age of 0.1 . To correct for the different strengths of correlations, we composited the aging score by using the weighted average of the variables, such that the Pearson correlations are used as weights. This score ensures that variables with a higher correlation with chronological age have a stronger effect on the composite aging score. The composite aging score $A_{x}$ of a certain person $\mathrm{X}$ is summarized by the equation

$$
A_{x}=\frac{\sum_{i=1}^{329} \frac{\operatorname{cov}\left(V_{i}, Y\right)}{\sigma_{V i} \cdot \sigma_{Y}} \cdot V_{i, x}}{\sum_{i=1}^{329} \frac{\operatorname{cov}\left(V_{i}, Y\right)}{\sigma_{V i} \cdot \sigma_{Y}}},
$$

where $V_{i, x}$ is the value of variable $i$ for subject $x$, and $\mathrm{Y}$ is the chronological ages of the subjects in the cohort.

For each chronological age, the composite aging scores $A$ of all subjects in the cohort at that age are averaged to provide a mean composite physiological aging score of each chronological age.

\section{Results}

Figure 1 shows the results of the PCA made of the six principal components described above. The error bars show the standard error of the score. As the figure shows, a relatively sharp change in physiological age can be noticed at around the age of 55 years. Then, a period of mild change in physiological age can be noticed until around the age of 65 years, when it changes noticeably. According to the analysis of the principal components, the progression is not linear and shows that physiological aging does not progress at a constant rate but has stages separated by short periods of more rapid aging.

Analysis of the different principal components separately shows largely similar results. Figure 2 shows the progression of the physical activity component. As the figure 
Fig. 2. Physical activity score and standard error of the mean of the physiological aging scores at different chronological ages.
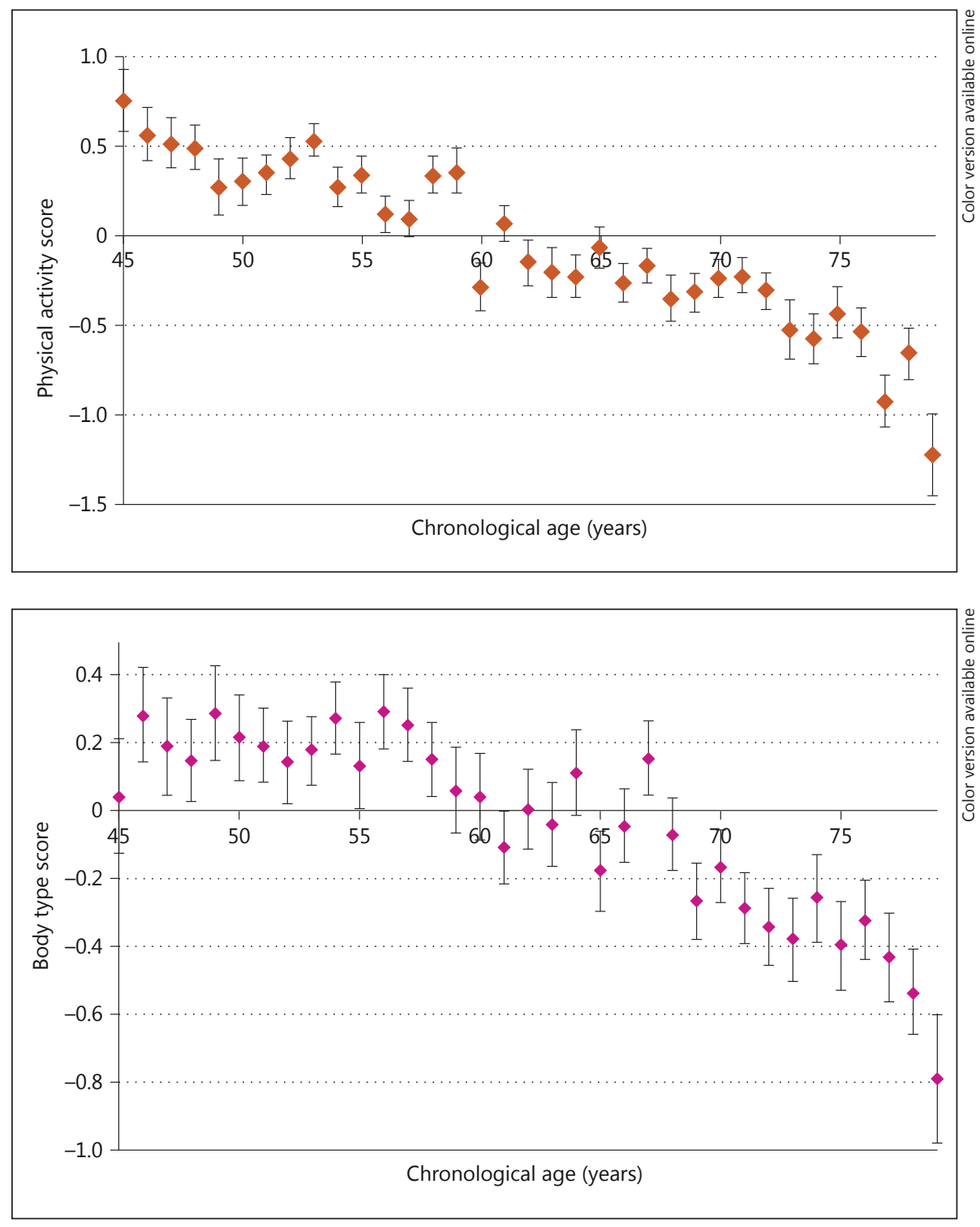

Fig. 3. Body type score and standard error of the mean of the physiological aging scores at different chronological ages. shows, a sharper decline is noticeable at around the age of 55 years, after which the progression stabilizes until after the chronological age of 70 years. Similar observations of rapid aging around the age of 55 years can also be seen in figures 3 and 4, displaying the aging score based on body type and heart health, respectively. Unlike the other components, the mental health score increases sharply after the age of 60 years but stays stable after the age of 65 years. The mental health component is displayed is figure 5 .

A similar profile of aging progression was observed also with the composite aging scores described above. Figure 6 shows how the composite aging score changes with chronological age. As the figure shows, the compos-

Aging Profile Analysis Using Comprehensive Sets of Variables ite aging score suggests that a period of slower aging before the age of 55 years is followed by more rapid aging between the ages of 55 and around 60 years, slowing again until increasing at around the age of 70 years. Except for the end of the eighth decade, the fastest progression of the composite aging score is observed at around the age of 55 years. Since, in this analysis, each composite aging score was based on 329 variables and the variables were weighted by their correlation with chronological age, the variations were much smaller compared to the PCA, and therefore the standard error was smaller.

As described in the section Design and Methods, we also applied PCA to the longitudinal data. Figure 7 
Fig. 4. Heart health score and standard error of the mean of the physiological aging scores at different chronological ages.

Fig. 5. Mental health score and standard error of the mean of the physiological aging scores at different chronological ages.

Fig. 6. The composite aging score and standard error as a function of chronological age.
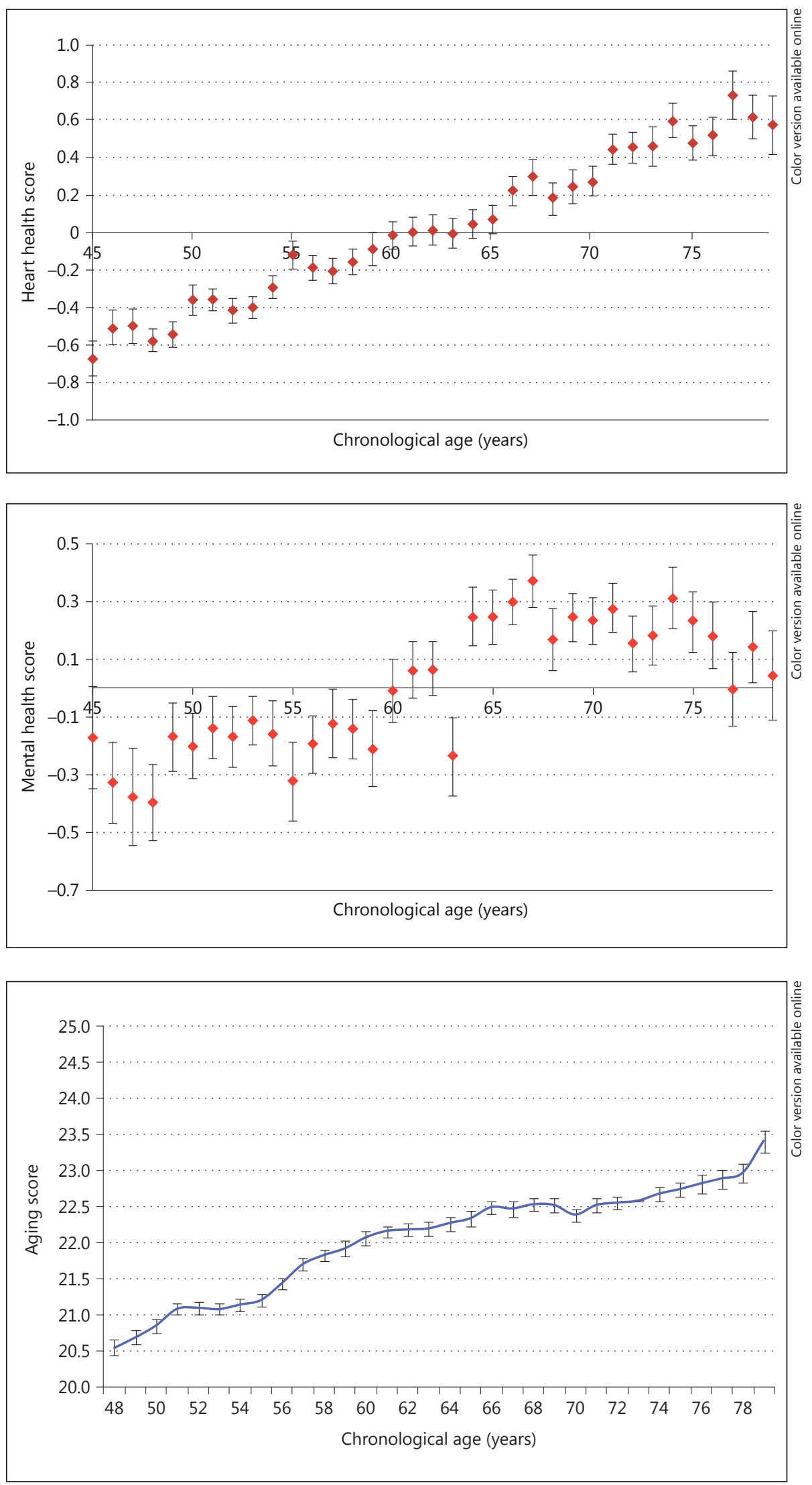


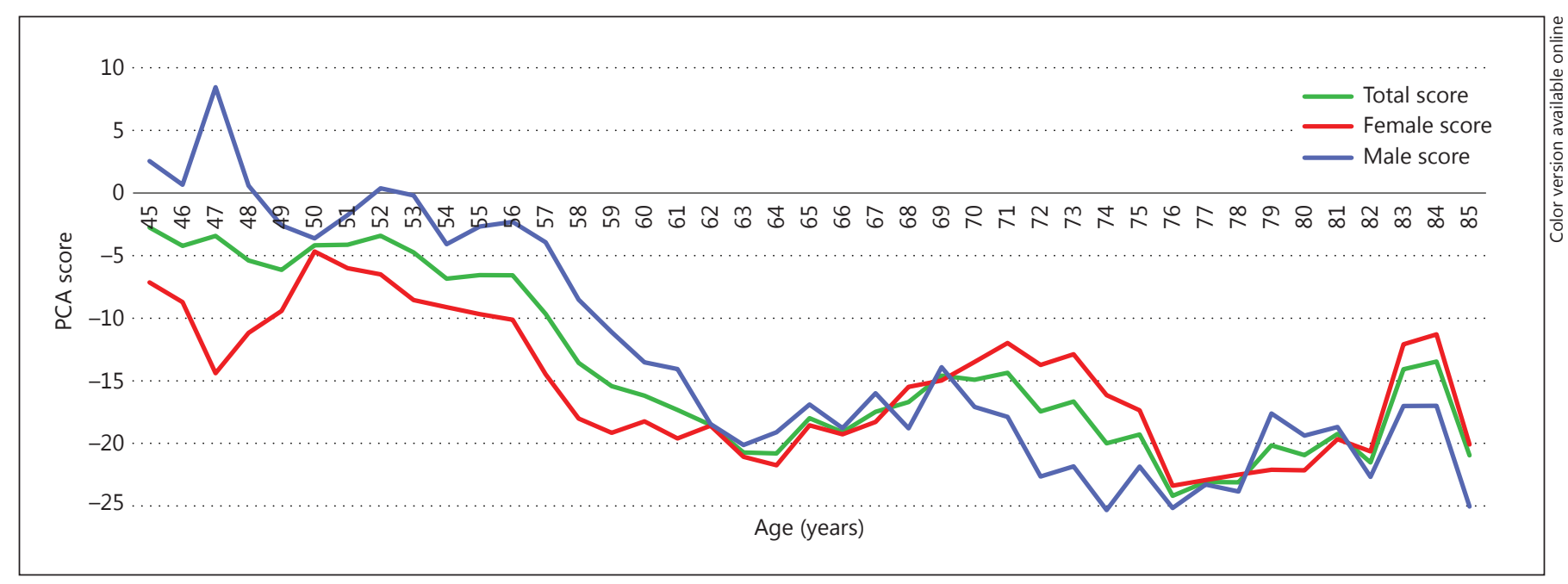

Fig. 7. Changes in physiological aging at different ages over a period of 72 months.

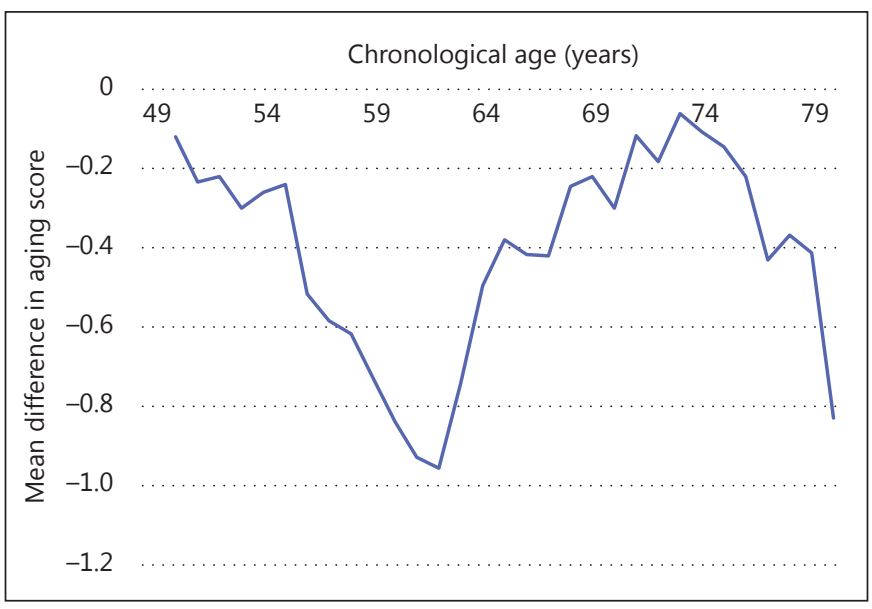

Fig. 8. Longitudinal analysis of the composite aging score at different ages.

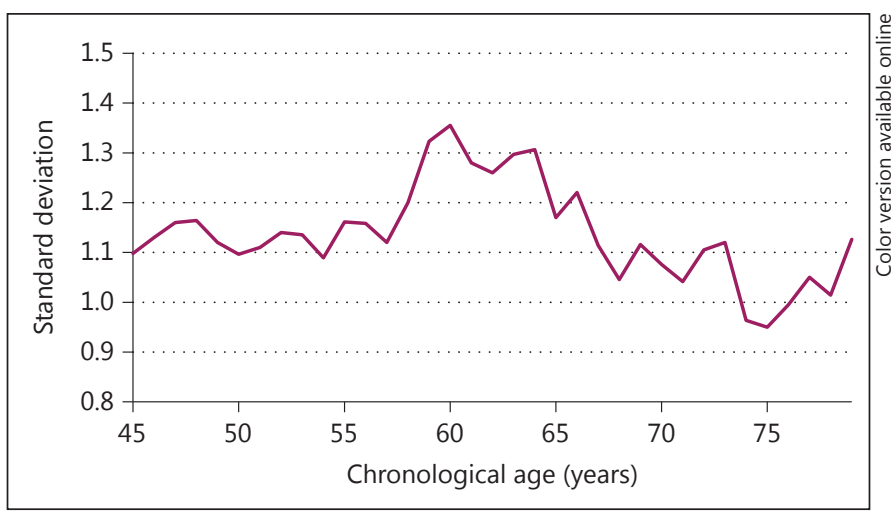

Fig. 9. Standard deviation of the physiological aging score at different ages.

Aging Profile Analysis Using Comprehensive Sets of Variables shows the PCA score for both men and women. As the figure shows, the longitudinal data generally show a decrease, but the sharpest decrease is observed when the measurements are taken before and after the age of 56 and until 62 years, indicating more rapid processes of aging around that age. Since the follow-up visit occurred 72 months after the baseline visit, and the age at the time of the visit was randomly distributed, rapid aging around the age of 55 years was expected to exhibit itself in the form of about 6 years of increased differences between the scores at the baseline and follow-up visits.

The longitudinal analysis of the composite aging score used in figure 6 and described in the section Design and Methods shows similar results. Figure 8 shows the average difference between the baseline and follow-up visits at different ages, such that the baseline visit was before that age and the follow-up visit was after. As the figure shows, between the ages of 56 and 62 years, the difference between the composite aging scores at the baseline and follow-up visits was the largest compared to other ages, showing accelerated aging during that age.

We also tested the distribution of the PCA aging scores at different ages. Figure 9 shows the standard deviation of the physiological aging scores. As the figure shows, the standard deviation decreases sharply after the age of 55 years and then gradually declines until around the age of 75 years. The gradual decrease in the standard deviation can also be the effect of a less heterogenic population in terms of their physiological aging state, since at the older ages, subjects who agreed to participate in the program in 
general might be in better physical shape. It can also be the effect of the increasing dominance of long-lived people, so that the homogeneity of the cohort increases as the weight of the long-lived subjects among the entire cohort increases.

\section{Discussion}

Although aging is a highly prevalent physiological process that affects almost all organisms, little is known about its driving mechanisms, and the complex nature of the numerous biological mechanisms related to aging makes aging difficult to measure. One contention is that aging is the process of irreparable accumulating damage, inheritance, and complex molecular processes [14] such as protein glycation [15], inflammation [16], and oxidative damage [17]. Assuming that aging is driven by irreparable accumulation of biomechanical junk, the effect of aging is stochastic, and therefore no reliable aging biomarker is expected.

However, this contention is challenged by the observation that some organisms such as Homaridae (lobsters) can live hundreds of years without showing any signs of age-related symptoms or physiological decline [18]. It should be mentioned that lobsters are very different from mammals and live in a very different environment. Studies with C. elegans also suggest that there is no evidence that environmental damage can lead to conditions associated with aging, such as chronic induction of stress responses [19].

Another approach is that the process of aging is driven by developmental pathways $[20,21]$. This paradigm has been supported by the observation that in C. elegans the expression of elt-5, which is responsible for the expression of a large number of age-regulated genes, changes during aging in a noncontinuous fashion [8]. These upregulations, observed around day 2 and day 8 of adulthood, are in agreement with the rapid alteration of the tissues [9].

While C. elegans is physiologically very different from mammals, this study shows that physiological indicators that correlate with chronological age do not necessarily progress linearly or exponentially and in fact feature several distinct stages separated by periods of rapid aging. These findings are in agreement with the contention proposed by Ferrucci et al. [22], according which human health shifts along a path of distinct states during aging. The results presented here, based on a cross-sectional and longitudinal analysis of a large number of physiological and environmental indicators, are also supported by the profile of alteration of human tissues, which shows the same periods of rapid aging around the age of 55 and after the age of 70 years [23]. These findings show that the process of aging might not be driven by the process of accumulation of irreparable damage alone, but that it can also be affected by age-regulating biological mechanisms.

Rapid aging after the age of 70 years was measured in subjects that lived beyond that age and who were also able to participate in the study. Therefore, this group of subjects might not be as randomly selected as the younger age groups. The expected slower aging of that group is not observed at the higher ages, but it is difficult to make conclusions about that group, since its aging profile cannot be compared to a control group due to the fact that subjects with lower longevity obviously do not provide data for these ages. However, it is also possible that the slower aging of long-lived people occurs during earlier ages. This can be tested by examining data collected throughout the adult life of the participants and also includes the longevity of each subject.

Strehler [24] proposed the aging criteria of cumulative, universal, progressive, intrinsic, and detrimental, later coined as the acronym CUPID [25]. However, the absence of a reliable single aging biomarker makes it difficult to quantitatively profile the process of aging. From an evolutionary perspective, longevity in humans should include the time required to mate, reproduce, and nurture children, that is, until they can reproduce and nurture their own children to continue the preservation of their species [26]. This period of time is defined as 'adaptive lifespan' [27], 'biological warranty period' [28], 'essential lifespan' [29], or 'longevity determination' [30, 31]. Given that aging can be driven by natural selection acting on genetic biological pathways [32], it can be expected that a rapid process of aging may be observed after that period. The findings in this paper are in agreement with previously proposed paradigms, according to which longevity until the age of 50-55 years is sufficient to obtain Darwinian fitness [28].

\section{Acknowledgements}

This research was supported in part by the Intramural Research Program of the NIH and the National Institute on Aging as well as by NSF grant No. 1157162 . We would also like to thank the reviewer, Bruce Carnes, for his numerous useful comments and discussions that helped to significantly improve the paper. 


\section{References}

1 Sprott RL: Biomarkers of aging and disease: introduction and definitions. Exp Gerontol 2010;45:2-4.

$>2$ Sprott RL: Biomarkers of aging. Gerontology 1999;54A:464-465.

3 Warner HR: Current status of efforts to measure and modulate the biological rate of aging. Gerontology 2004;59A:692-696.

4 Curb JD, Ceria-Ulep CD, Rodriguez BL, Grove J, Guralnik J, Willcox BJ, Donlon TA, Masaki KH, Chen R: Performance-based measures of physical function for high-function populations. J Am Geriatr Soc 2006;54: 737-742.

$\checkmark 5$ Rantanen T, Masaki KH, He Q, Ross GW, Willcox BJ, White L: Midlife muscle strength and human longevity up to age 100 years: a 44-year prospective study among a decedent cohort. Age (Dordr) 2012;34:563-570.

6 Bell CL, Chen R, Masaki KH, Yee P, He Q, Grove J, Donlon T, Curb JD, Willcox DC, Poon LW, Willcox BJ: Late-life factors associated with healthy aging in older men. J Am Geriatr Soc 2014;62:880-888.

7 Ma X, Becker D, Arena VC, Vicini P, Greenbaum C: The effect of age on insulin sensitivity and insulin secretion on first-degree relatives of type 1 diabetic patients: a population analysis. J Clin Endocrinol Metab 2009;94 2446-2451.

-8 Budovskaya YV, Wu K, Southworth LK, Jiang M, Tedesco P, Johnson TE, Kim SK: An elt-3/ elt-5/elt-6 GATA transcription circuit guides aging in C. elegans. Cell 2008;134:291-303.

9 Shamir L, Wolkow C, Goldberg IG: Quantitative measurement of aging using image texture entropy. Bioinformatics 2009;25:30603063.
10 Lester G: Clinical research in OA - the NIH Osteoarthritis Initiative. J Musculoskelet Neuronal Interact 2008;8:313-314.

$>11$ Julliffe I: Principal Component Analysis. Hoboken, John Wiley \& Sons, 2005.

12 Kulminski AM, Ukraintseva SV, Culminskaya IV, Arbeev KG, Land KC, Akushevich L, Yashin AI: Cumulative deficits and physiological indices as predictors of mortality and long life. J Gerontol A Biol Sci 2008;63:10531059.

13 Carpenter S, Huffman K: Visualizing Psychology, ed 3. New York, Wiley, 2012.

14 Yin D, Chen K: The essential mechanisms of aging: irreparable damage accumulation of biochemical side-reactions. Exp Gerontol 2005;40:455-465.

15 Ulrich P, Cerami A: Protein glycation, diabetes, and aging. Recent Prog Horm Res 2001; 56:1-21.

16 McGeer PL, McGeer EG: Inflammation and the degenerative disease of aging. Ann NY Acad Sci 2004;1035:104-116.

-17 Lu T, Pan Y, Kao SY, Li C, Kohane I, Chan J, Yankner BA: Gene regulation and DNA damage in the ageing human brain. Nature 2004; 429:883-891.

18 Guerin JC: Emerging area of aging research: long-lived animals with negligible senescence. Ann NY Acad Sci 2007;1019:518-520.

19 Lund J, Tedesco P, Duke K, Wang J, Kim SK, Johnson TE: Transcriptional profile of aging in C. elegans. Curr Biol 2002;12:1566-1573.

20 Williams GC: Pleiotropy, natural selection, and the evolution of senescence. Evolution 1957;11:398-411
21 Kirkwood TB, Rose MR: Evolution of senescence: late survival sacrificed for reproduction. Philos Trans R Soc Lond B Biol Sci 1991; 332:15-24.

22 Ferrucci L, Giallauria F, Schlessinger D: Mapping the road to resilience: novel math for the study of frailty. Mech Ageing Dev 2008;129: 677-679.

23 Shamir L: Quantitative measurement of human aging using computer-aided radiographic texture analysis. Comput Methods Biomech Biomed Eng Imaging Vis 2013;1:175183

24 Strehler BL: Origin and comparison of the ef fects of time and high-energy radiations on living systems. Q Rev Biol 1959;34:117-142.

25 Arking R: Biology of Aging: Observations and Principles. New York, Oxford University Press, 2006.

26 Carnes BA, Olshansky J, Grahn D: Biological evidence for limits to the duration of life. Biogerontology 2003;4:31-45.

27 Gurven M, Hilliard K: Longevity among hunter-gatherers: a cross-cultural examination. Pop Dev Rev 2007;33:321-365.

28 Carnes BA, Witten TM: How long must humans live? J Gerontol A Biol Sci Med Sci 2014; 69:965-970.

29 Rattan SI: Biogerontology: the next step. Ann NY Acad Sci 2000;908:282-290.

30 Hayflick L: Biological aging is no longer an unsolved problem. Ann NY Acad Sci 2007; 1100:1-13.

31 Hayflick L: The future of ageing. Nature 2000; 408:267-269.

32 Sacher GA: Longevity and aging in vertebrate evolution. Bioscience 1978;28:497-501. 